\section{G582 PHASE 3 RANDOMISED CONTROLLED TRIAL OF VITAMIN D SUPPLEMENTATION IN 8,851 MONGOLIAN SCHOOLCHILDREN}

${ }^{1,2} \mathrm{D}$ Ganmaa, ${ }^{2} \mathrm{G}$ Gantsetseg, ${ }^{2} \mathrm{D}$ Khulan, ${ }^{2} \mathrm{~S}$ Ariunzaya, ${ }^{2} \mathrm{~B}$ Delgereh, ${ }^{2} \mathrm{E}$ Sumiya, ${ }^{2} B$ Amarsaikhan, ${ }^{3}$ AR Martineau. ${ }^{1}$ Harvard T.H. Chan School of Public Health, Harvard University, Boston, USA; ${ }^{2}$ Mongolian Health Initiative, Mongolian National University of Medical Sciences, Ulaanbaatar, Mongolia; ${ }^{3}$ Barts and The London School of Medicine and Dentistry, QMUL, London, UK

\subsection{6/archdischild-2020-rcpch.499}

Aims To determine the effects of vitamin D supplementation on diverse health outcomes in vitamin D-deficient schoolchildren. Methods We performed a phase 3 randomised placebo-controlled clinical trial in Ulaanbaatar, Mongolia, to determine whether weekly oral supplementation with 14,000 IU vitamin $\mathrm{D}_{3}$, administered for 3 years, reduced acquisition of tuberculosis infection in 8,851 schoolchildren aged 6 to 13 years (primary outcome). Secondary efficacy outcomes evaluated in all study participants included incidence of active tuberculosis, acute respiratory infections, atopic diseases and fractures, and growth and muscle strength. Sub-studies evaluated effects of the intervention on bone mineral density, spirometry and pubertal development $(n=1,465)$, physical fitness $(n=615)$ and examination performance $(n=2,097)$. Safety outcomes included mortality and incidence of serious adverse events. Sub-group analyses were conducted for all efficacy outcomes to determine whether the effect of the intervention varied according to baseline 25-hydroxyvitamin D levels.

Results Of the 8,851 randomized participants, 95.6\% had vita$\min \mathrm{D}$ deficiency $(25[\mathrm{OH}] \mathrm{D}<20 \mathrm{ng} / \mathrm{mL})$ at baseline, and 91.7\% completed the study. Mean end-study 25(OH)D levels were 29.8 vs. $9.7 \mathrm{ng} / \mathrm{mL}$ in children randomized to intervention vs. placebo $(\mathrm{p}<0.001)$. Vitamin $\mathrm{D}$ supplementation did not influence the risk of QuantiFERON-TB Gold conversion $(\mathrm{P}=0.42)$, incident active tuberculosis $(\mathrm{P}=0.63)$, incident asthma $(\mathrm{P}=0.32)$, incident atopic dermatitis $(\mathrm{P}=0.39)$, incident allergic rhinitis $(\mathrm{P}=0.45)$, proportion of children experiencing one or more fractures $(\mathrm{P}=0.65)$, mean height-for-age $\mathrm{z}$-score (0.84), mean grip strength $(\mathrm{P}=0.77)$, mean bone mineral density at the radius $(\mathrm{P}=0.14)$, mean Tanner scores for pubertal development $(\mathrm{P} \geq 0.28)$, mean forced expiratory volume in 1 second (FEV1, $\mathrm{P}=0.35$ ), mean forced vital capacity (FVC, $\mathrm{P}=0.95)$, mean maximal oxygen consumption during a 20 metre shuttle run $(\mathrm{VO} 2 \mathrm{max}, \mathrm{P}=0.22)$ or mean end-of-year mathematics exam scores $(\mathrm{P}=0.57)$. Effects of the intervention did not differ according to baseline 25-hydroxyvitamin D levels $(<10 \mathrm{ng} / \mathrm{mL}$ vs. $\geq 10 \mathrm{ng} / \mathrm{mL}$ ) for any efficacy outcome studied. Neither mortality nor incidence of serious adverse events differed between study arms.

Conclusions A weekly oral dose of 14,000 IU vitamin $D_{3}$, administered for 3 years, was safe and effective in elevating 25 -hydroxyvitamin $\mathrm{D}$ levels into the high physiological range in vitamin D-deficient schoolchildren, but it did not impact any efficacy outcome investigated.

\section{G583 SPARE PENS IN SCHOOLS: A SURVEY AUDITING THE UPTAKE OF THE SPARE PENS IN SCHOOL SCHEME IN PETERBOROUGH}

AC Barker, E Wawrzkowicz. Paediatrics, Peterborough City Hospital, North West Anglia Trust, Peterborough, UK

10.1136/archdischild-2020-rcpch.500
Introduction Analphylaxis is a severe and often sudden allergic reaction which can be fatal. Up to $8 \%$ of children in the UK have a food allergy and on average most school classes will have 1 or 2 children per class with allergies. In the UK $17 \%$ of fatal allergic reactions happened in school which is the driving force behind the spare pens in schools scheme. On the 1st October 2017 the human medicines (amendment) regulations 2017, allowed schools to obtain without a prescription 'spare' adrenaline auto injector (AAI) devices, for use in emergencies. The audit objective was to gauge the uptake of the spare pens in school scheme and to identify potential barriers obstructing uptake.

Method We designed a survey and sent it to 74 schools in Peterborough. We had replies from 11 schools (14\%) capturing a total of 6231 students.

Results 5 (45\%) schools had heard of the spare pens in school scheme, however only 2 (18\%) schools had utilised it. All schools stated they were able to treat anaphylaxis with an AAI device either immediately or within minutes and 5 of the schools $(45 \%)$ all staff were trained to use AAI devices. We were pleased to hear that $90 \%$ of the respondents had allergy action plans in place.

Conclusions The most common reason cited for not utilising the spare pens in schools scheme was the cost of the pens. Pharmacies charge for the cost of the AAI device and may add a handling charge. Unfortunately, at present there are no funds available to help schools cover this cost. As a direct consequence of this audit we are currently in talks with the pharmacy lead for CCG regarding the possibility of mobilising funds to help schools buy spare pens. Our specialist allergy nurse will encourage spare pens uptake at an annual training event planned with school nurses. We will re-audit next year to hopefully demonstrate an improved uptake. We are aware that the audit generated a small sample size and may be subject to bias as the schools who replied could represent the most engaged and up to date schools in Peterborough.

\section{G584 AN AUDIT OF THE EMERGENCY MANAGEMENT OF ANAPHYLAXIS IN CHILDREN IN SECONDARY CARE}

A Stratford, V Sharma, A Ali, L Ventilacion. Paediatrics, Lister Hospital, Stevenage, UK

\subsection{6/archdischild-2020-rcpch.501}

Aim/Objective There is an increasing incidence in anaphylaxis reported globally and in the UK. Due to this, there is raised anxiety in our local population around the management of anaphylaxis, especially in light of the shortage of adrenaline auto-injector pens last year.

The aim of this audit project is to assess the management of paediatric patients presenting with anaphylaxis to the Paediatrics Emergency Department (PED) and Children's assessment Unit (CAU) and whether they are appropriately managed in accordance with current NICE and local guidelines.

Methods We reviewed the notes of patients who present to PED and CAU from June 2018 until January 2019. Patients were identified through search words such as 'anaphylaxis' and 'allergic reaction' in coding. The notes were reviewed and data collected using the Anaphylaxis Audit Proforma. 
Results 26 patients were identified based on the keyword search. 6/26 patients (19 months - 15 years) fulfilled the diagnostic criteria of anaphylaxis; however, only $2 / 6$ had clearly documented diagnosis of anaphylaxis. All 6 were treated with Chlorphenamine; 4/6 had IM adrenaline; and 4/ 6 had IV Hydrocortisone. 3/6 patients did not have an identifiable allergen trigger, all of whom did not have a tryptase level taken. 5/6 had minimum recommended time of 6 hours of observation. At discharge, 4/6 patients had an auto-injector prescribed and training provided. All patients had documented safety-advice given, with an anaphylaxis action plan provided to $4 / 6$. Overall, specific documentation pertaining to information on discharge, including risk of biphasic reaction (1/6), was poor. Reassuringly, all patients were referred to the allergy clinic.

Conclusions This audit identifies the need for further education on recognition, correct diagnosis and management of anaphylaxis, with emphasis on clear documentation especially of safety-net information given at discharge. Promoting use of the Anaphylaxis Proforma and guideline aims to improve safety and standardise the care of patients presenting with anaphylaxis.

\section{G585(P) SUPERVISED FEEDS: A REVIEW OF LOCAL PRACTICE}

CE Baker, A Aston, N Patel. Paediatric Allergy, Royal London Hospital, Barts Health NHS Trust, London, UK

\subsection{6/archdischild-2020-rcpch.502}

Background Food allergy is becoming increasingly common in the paediatric population resulting in increased demand for allergy services. Food allergy investigations can include inhospital oral food challenges and supervised feeds. In 2018 supervised feeds were introduced in this hospital for challenges deemed as lower risk. This involves consuming a predetermined amount of food within 30 minutes and being observed for an hour thereafter. An oral food challenge involves food given in incremental doses with observations in-between and 2 hours after, therefore, it is a longer process.

Aims To review current departmental practice of supervised feeds focussing on the following:

1. What patterns of food are being tested?

2. What were the skin prick test (SPT)/specific IgE results?

3. Explore test failures in further detail.

Method A retrospective analysis of supervised feeds recorded in the unit database from May 2018 to May 2019. If data was missing then discharge summaries from the admission were reviewed.

Results The number of supervised feeds recorded on the database between May 2018-2019 totalled 162, 156 of these were included. The most common foods tested were tree nuts $(30 \%)$ followed by peanuts (20\%). SPT results ranged from 0-4 mm. 2 patients' had results higher than recommended by current guidelines, however, both passed their supervised feed. Overall failure rate was $13.5 \%$. SPT was 0 $\mathrm{mm}$ in $64 \%$ of patients and $11 \%$ of these failed. Reasons for failure included refusal to eat (4 patients) and allergic reaction; ranging from itching to anaphylaxis (1 patient). Treatment required ranged from observation, antihistamine, to IM adrenaline (1patient).
Conclusion Supervised feeds are a useful method to assessing food allergy in appropriately selected patients. Overall failure rate of supervised feeds in this study was low at $13.5 \% .10$ patients had a SPT of $0 \mathrm{~mm}$ and failed, so it is important to remember even though deemed low risk there is still a risk of significant reaction. By identifying appropriate patients as suitable for supervised feed then their time spent in hospital can be reduced and may allow for an increased number of in-hospital challenges throughout the year.

\section{G586(P) CASE REPORT OF HYPER IGM SYNDROME IN DOWN SYNDROME CHILD}

MC Quek, A Migowa, RW Gitakah. Department of Paediatrics and Child Health, Aga Khan University Hospital, Nairobi, Kenya

\subsection{6/archdischild-2020-rcpch.503}

Introduction Down syndrome is the most common genetic disease that often presents with a high frequency of infections especially upper airway infections in their early years, characterized by increased severity and prolonged course of disease. Individuals with Down syndrome are known to have abnormal function of the B-cell function, which might present with high $\mathrm{IgG}$ and low $\operatorname{IgM}$ serum level.

Case Description A one-year-old known Down syndrome child presented with persistent nasal congestion, fever, noisy breathing, and daily cough for 8 months. He had several outpatient consults and treatment with antibiotics but with poor relief of symptoms. Due to persistent symptoms, he was referred to the ENT surgeon. His paranasal radiographs revealed sinusitis. He was then referred to a pediatric infectious specialist for further management. Examination revealed a sick-looking, syndromic child who was having marked nasal congestion, global hypotonia and generalized erythematous rash. He was well-nourished. Serum immunoglobulin test show marked elevated Ig $\mathrm{M}$ with low Ig G and Ig A levels. Rheumatologist agreed with the possible diagnosis of primary immunodeficiency syndrome (PIDS). Pulmonologist confirmed no underlying congenital lung pathology from the chest CT scan. The echocardiogram was unremarkable. He received intravenous immunoglobulin (IVIG) as part of the therapy and his symptoms improved markedly. An impression of Hyper Ig $M$ syndrome was made. He received two other courses of IVIG after discharge. Currently, the mother reports good improvement of symptoms.

Discussion Hyper IgM syndrome is a primary immunodeficiency syndrome characterized by defective CD 40 signaling, which leads to a defect in Ig class switching recombination.

The association of Down syndrome with PIDS is not clearly demonstrated. In our case, elevated Ig $\mathrm{M}$ was an incidental finding. Warning signs present in children with Down syndrome warrants a detailed immunology investigation to exclude primary immunodeficiency. Diagnosing PIDS imposed a great challenge to doctors especially due to the co-existing condition. A delay in diagnosis is common and associated with increased morbidity and parental distress.

Conclusion There is a need to create awareness amongst the healthcare workers concerning PIDS especially in children with co-existing diseases. We need to assure the proper diagnosis and proper care management are provided to the affected children. 